\title{
Large-field high-resolution two-photon digital scanned light-sheet microscopy
}

Cell Research (2015) 25:254-257. doi:10.1038/cr.2014.124; published online 26 September 2014

\section{Dear Editor,}

Recent advent of light-sheet fluorescent microscopy (LSFM) has revolutionized three-dimensional biological imaging with high temporal resolution and minimal photodamage, enabling long-term fluorescence imaging of tissues and small organisms [1-2]. By combining two-photon fluorescence excitation with LSFM, Truong et al. [3] have created a two-photon digital scanned lightsheet microscope (2P-DSLM), allowing for deep-tissue imaging of highly scattering Drosophila embryos and fast beating hearts of zebrafish. Similar to classical LSFM configurations, a 2P-DSLM uses a low numerical aperture $(\mathrm{NA}<0.1)$ to achieve a long and homogenous illumination. This leads to a thick light sheet and thus reduces axial resolution and image contrast. On the other hand, Betzig and colleagues have used a Bessel beam to generate thin single-photon light sheet that yields superb axial resolution [4]. However, the field of view and the penetration depth are limited in such system.

To achieve high axial resolution (thin light sheet) and large field of view simultaneously, here we have developed a novel two-photon three-axis digital scanned lightsheet microscope (2P3A-DSLM) based on ultrafast axial scanning of illumination focal spot with a tunable acoustic gradient (TAG) index device. This new technique provides a sub-micron axial resolution, a large field of view of $170 \times 170 \mu^{2}$ in deep tissues, and a high temporal resolution limited only by the detection camera. In vivo imaging with 2P3A-DSLM resolved subcellular structures and dynamic processes in small organisms.

A schematic illustration and configuration of the 2P3A-DSLM is shown in Figure 1A. A TAG lens uses acoustic wave to radially excite a fluid-filled cylindrical cavity and produce continuous changes in refractive power that enables rapid change of its axial focal plane within $10 \mu$ s [5-6]. Here we used the TAG lens to rapidly translate the focal spot of a femtosecond laser (140 fs, repetition rate, $80 \mathrm{MHz}$, Chameleon Version II, Coherent) along the optics axis ( $x$-direction) in front of the illumination objective ( $40 \times$ NA 0.8 , Nikon). A gal- vo scanning mirror (GSM) was used to scan the laser beam vertically to the axis of the illumination objective (y-direction). By driving the TAG lens to refocus at $\sim 450$ $\mathrm{kHz}$ and the GSM at $1 \mathrm{kHz}$, we were able to create an ultra-thin scanned 2P light sheet within one millisecond. The size of the scanned light sheet can be modulated by adjusting the modulation intensity of the TAG ( $x$-direction) and scanning amplitude of the galvo mirrors ( $y$-direction) (Figure 1B-1D). During the fast axial scanning of the TAG lens, the sinusoidal motion of the focal spot along axial position and the change of illumination NA result in the uneven illumination of the light sheet. To compensate the unevenness, we used an ultra-fast Pockels cell (Conoptics, 350-160LA) to dynamically adjust illumination power and effectively remove non-uniform illumination along the axial axis (Supplementary information, Figure S1A-S1H).

We quantified the performance of our system by imaging FITC solution (Figure 1B-1F and Supplementary information, Figure S1A-S1H). The depth of field (DOF) along $x$-direction was found to linearly increase up to $170 \mu \mathrm{m}$ when the modulation intensity of the TAG was increased to $35 \%$ (Figure 1E). The light-sheet thickness at the beginning and in the middle remained nearly constant below $1 \mu \mathrm{m}$ while the DOF varied between 5 and $170 \mu \mathrm{m}$ (Figure 1F and Supplementary information, Figure S1B-S1E). At 35\% TAG modulation intensity, the thickness of the light sheet at the far-end of the 170 $\mu \mathrm{m}$ DOF was increased to $1.5 \mu \mathrm{m}$, still less than that of common LSM configurations $(2 \sim 8 \mu \mathrm{m})$ (Figure $1 \mathrm{~F}$ and Supplementary information, Figure S1D) [7]. Because the infinity-correction range of the illumination objective is limited, the light sheet abruptly expanded in $z$-direction at TAG modulation intensity $>35 \%$ (Supplementary information, Figure S1E). Larger tunable range of DOF could be obtained by using an illumination objective with low NA and magnification (Supplementary information, Figure S1I-S1K), but it would increase the thickness of light sheet and sacrifice the spatial resolution of the system (Supplementary information, Figure S1L).

We evaluated the axial ( $y-z$ plane $)$ and lateral $(x-y$ 

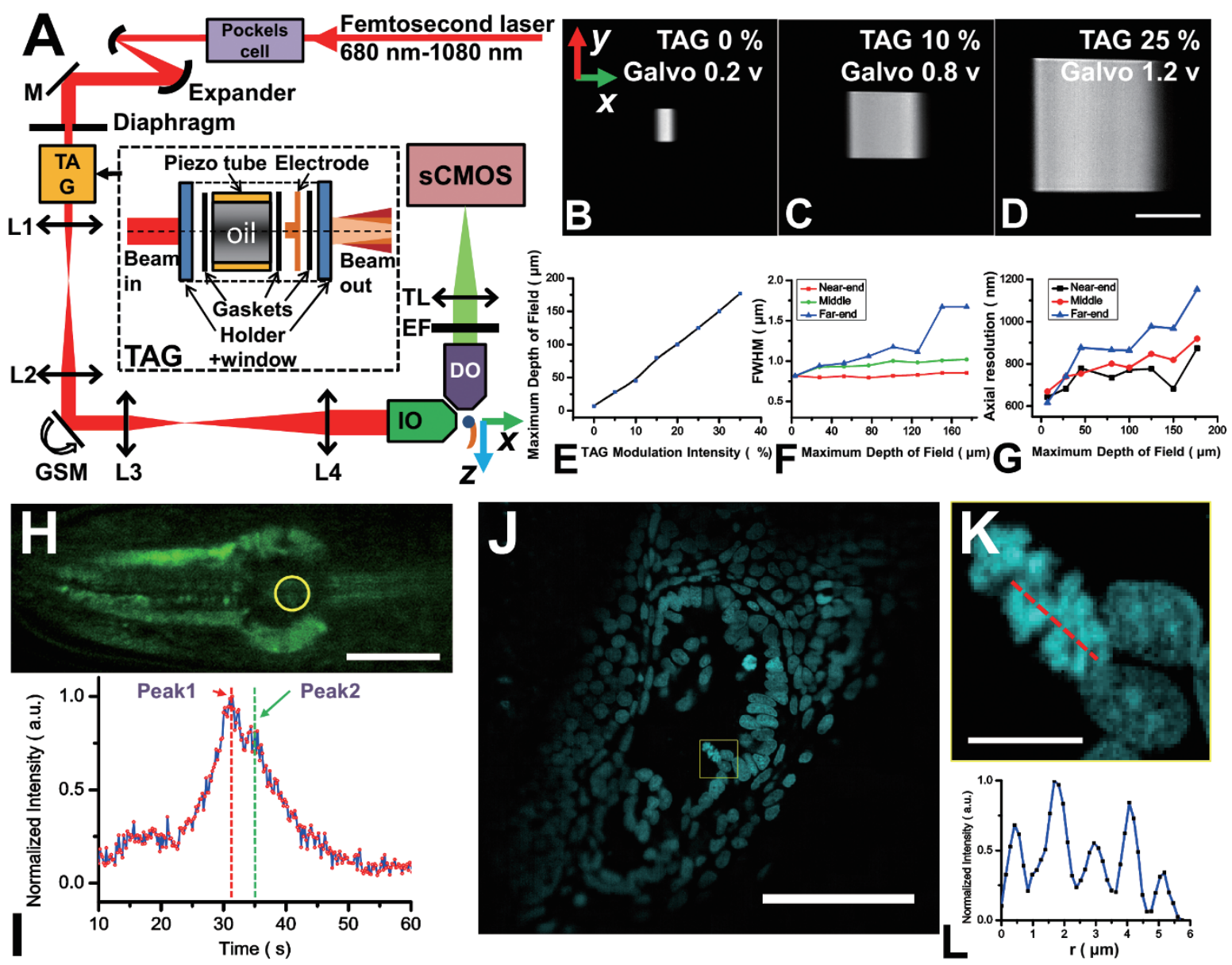

Figure 1 (A) A schematic illustration of 2P3A-DSLM. We used a commercial Ti-sapphire femtosecond laser (Chameleon Version II, Coherent) as the illumination light source, and expanded the beam by a pair of concave mirrors. The focal length of the TAG lens was modulated sinusoidally at frequency of $100-500 \mathrm{kHz}$ (diopter, \pm 30 ), which rapidly scanned the laser focus along the optics axis ( $x$-direction) in front of the illumination objective (IO, 40× NA 0.8, Nikon). The GSM scanned the laser beam vertically to the illumination objective ( $y$-direction). The TAG and the GSM were positioned at two conjugate planes of the back focal plane (BFP) of the illumination objective by L1 $(50 \mathrm{~mm}), \mathrm{L} 2(100 \mathrm{~mm}), \mathrm{L} 3(100 \mathrm{~mm})$ and L4 (200 mm). This configuration minimized the distortion and divergence of illumination intensity during scanning. The detection objective (DO, 40× NA 0.8, Nikon), emission filter, tube lens $(200 \mathrm{~mm}$ ) and sCMOS camera (flash 4.0, $2048 \times 2$ 048, Hamamatsu) were carefully aligned to record fluorescence images. A USB-controlled 3D stage (Thorlabs, MT3-Z8) combined with a fast Piezo Z-stepper (Edmund, Nanopositioning Piezo) was used to translate the sample for fast 3D imaging. The structure of a TAG len is given in the inset [5, 6]. EF, emission filter; TL, tunable lens. (B-D) Tailorable light sheet size from less than $10 \times 10 \mu \mathrm{m}^{2}$ to 170 $\times 170 \mu \mathrm{m}^{2}$ driven by intensity modulation of the TAG. (E) The DOF was linearly related with the increase of TAG modulation intensity. (F) The thickness of the light sheet at the near-end and the middle remained nearly constant below $1 \mu \mathrm{m}$, while the thickness at the far-end increased to $1.5 \mu \mathrm{m}$ when DOF reached $170 \mu \mathrm{m}$. (G) The axial resolution is below $1 \mu \mathrm{m}$ under all circumstances except at the far-end of the $170 \mu \mathrm{m}$ DOF (TAG modulation, 35\%). (H-I) Dynamic imaging of mitochondria in live mt-cpYFP transgenic $C$. elegans pharynx on adult day 3 (also refer to Supplementary information, Movie S1) revealed fast split in the mitoflash fluorescence trace. (J-L) 2P3A-DSLM 3D imaging of DAPI-labeled $(2 \mu \mathrm{g} / \mathrm{ml})$ nuclei in the heart of a live zebrafish. (J) A selected section from the Z-stack movie (Supplementary information, Movie S3); (K) Selected area in J shows cells in division phase and inter-phase. Dividing chromosomes within single nucleus were clearly resolved; (L) The fluorescence profile of the red line imposed on the chromosome shown in K. Images in H, J and K were deconvolved using Image J RL algorithm. Scale bar: $100 \mu \mathrm{m}$ (B-D), $20 \mu \mathrm{m}$ (H), $50 \mu \mathrm{m}$ (J) and 5 $\mu \mathrm{m}(\mathbf{K})$.

plane) resolution of the system at different TAG modulations by measuring fluorescence beads embedded in agarose gel (Figure 1G and Supplementary information, Figure S1M-S1N). The lateral FWHM of beads ranged 
between $420 \mathrm{~nm}$ and $450 \mathrm{~nm}$ at different TAG modulation intensities in the whole excitation field, close to the Abbe limit (Supplementary information, Figure S1MS1N). The axial FWHM of beads at the near-end and the middle of the excitation field were maintained around $700 \sim 800 \mathrm{~nm}$ when TAG modulation changed from $0 \%$ to $35 \%$ (Figure $1 \mathrm{G}$ ). The axial resolution at the far-end of the excitation field was kept below $1 \mu \mathrm{m}$ except at TAG modulation of $35 \%$ (Figure $1 \mathrm{G}$ ). These experimental data agree with the theoretical analysis (Supplementary information, Figure S1L), indicating that our system provides better resolution than conventional light-sheet microscopy and even two-photon laser scanning microscopy (2P-LSM) with a higher NA objective [3].

Next we tested its performance in biological studies by imaging subcellular structures and dynamics in vivo. Mitochondria are pivotal membrane-bound organelles in eukaryotic cells that range from 0.5 to $1 \mu \mathrm{m}$ in diameter. Single mitochondrion produces transient superoxide bursts known as "mitochondrial flashes" (mitoflashes) in live cells [8-9]. Here we monitored time-lapse dynamics of mitochondria in the pharynx of mt-cpYFP transgenic $C$. elegans on adult day 3 (Figure $1 \mathrm{H}$ and Supplementary information, Movie S1). With images of $2048 \times 2048$ pixels recorded at $5 \mathrm{~Hz}$ frame rate, we were able to resolve splits in mitoflash peaks during its progression (Figure 1I). At $820 \mathrm{~Hz}$ frame rate with $2048 \times 256$ pixels frame size, we succeeded in imaging "calcium nanosparks" in the dyadic space of a single cultured mouse cardiac myocyte [10] (Supplementary information, Figure S1O-S1Q and Movie S2).

Due to the small size of chromosomes, resolving chromosomal segregation during cell division in cultured single cells or at the earlier stage of embryo development usually requires high-NA, high-magnification objectives. Using 2P3A-DSLM, we were able to visualize hundreds of nuclei in the heart of a day 3 zebrafish simultaneously. Chromosome structures from a few dividing cells could be well resolved due to the extremely thin light sheet, low background and high contrast provided by the new imaging method (Figure 1J, 1K and Supplementary information, Movie S3).

Low light exposure is pivotal for the long-term imaging of biological samples. By continuous three-dimensional imaging of mt-cpYFP transgenic C. elegans, we compared the photobleaching of the 2P3A-DSLM system with a conventional 2P-LSM system. No significant bleaching was visible in our system after $15 \mathrm{~min}$ of recording, which is in sharp contrast to $>50 \%$ bleaching on the 2P-LSM setup (Supplementary information, Figure S1R-S1X and Movie S4). This is mainly due to the fact that for the same PSF axial stretch (Supplementary information, Figure S1R) and signal rate, the 2P3ADSLM requires a lower illumination NA, thus reducing the peak intensity of the excitation light and minimizing the supra-quadratic photodamage (Supplementary information, Figure S1R-S1X) [3]. In addition, we did theoretical comparison of the photodamage among 2P3ADSLM, 2P-DSLM and static light-sheet microscopy. For the same signal rate, 2P3A-DSLM shows lower linear photodamage, equal quadratic photodamage, and higher supra-quadratic photodamage, compared with 2P-DSLM and static light-sheet microscopy (Supplementary information, Figure S1Y).

In summary, our newly developed 2P3A-DSLM combines large field of view, minimal photobleaching, and high axial and temporal resolution with deep-tissue imaging. We demonstrate its superior performance by resolving subcellular structures and tracking single-mitochondrion dynamic processes in live model organisms.

\section{Acknowledgments}

We thank Fuzeng Niu for his help on the optics, Liwa Shao and Fujian Lu for biological experiments, Yongxiao Li for the Labview programming, and Yi Wu for the help on some of the data analysis. $\operatorname{Tg}(k d r l: E G F P)$ fish is a gift from Jingwei Xiong at Peking University. The work is supported by grants from the Major State Basic Research Program of China (2013CB531200, 2011CB809100 and 2012CB518200), the National Key Technology R\&D Program (SQ2011SF11B01041), the National Natural Science Foundation of China (31327901, 31221002, 81222020 and 21390410), and the Beijing Natural Science Foundation (7121008).

Weijian Zong ${ }^{1,2}$, Jia Zhao ${ }^{1}$, Xuanyang Chen ${ }^{1}$, Yuan Lin ${ }^{1}$, Huixia Ren ${ }^{1}$, Yunfeng Zhang ${ }^{4}$, Ming Fan ${ }^{2}$, Zhuan Zhou ${ }^{1}$, Heping Cheng ${ }^{1}$, Yujie Sun ${ }^{3}$, Liangyi Chen

\footnotetext{
${ }^{I}$ The State Key Laboratory of Biomembrane and Membrane Biotechnology, Institute of Molecular Medicine, Beijing Key Laboratory of Cardiometabolic Molecular Medicine, Institute of Molecular Medicine, Peking-Tsinghua Center for Life Sciences, Peking University, Beijing 100871, China; ${ }^{2}$ China Department of Cognitive Sciences, Institute of Basic Medical Sciences, Beijing 100850, China; ${ }^{3}$ Biodynamic Optical Imaging Center, Peking University, Beijing 100871, China; ${ }^{4}$ School of Electronics Engineering and Computer Science, Peking University, Beijing 100871, China Correspondences: Liangyi Chen ${ }^{\mathrm{a}}$, Yujie Sun ${ }^{\mathrm{b}}$

${ }^{a}$ Tel: +86-10-62764959

E-mail: lychen@pku.edu.cn

bE-mail: sun_yujie@pku.edu.cn
}

\section{References}

1 Pampaloni F, Reynaud EG, Stelzer EH. Nat Rev Mol Cell Biol 2007; 8:839-845.

2 Keller PJ, Schmidt AD, Wittbrodt J, et al. Science 2008; 322:10651069. 
3 Truong TV, Supatto W, Koos DS, et al. Nat Methods 2011; 8:757-760. Gao L, Shao L, Higgins CD, et al. Cell 2012; 151:1370-1385.

McLeod E, Arnold CB. Appl Opt 2008; 47:3609-3618.

6 Olivier N, Mermillod-Blondin A, Arnold CB, et al. Opt Lett 2009; 34:1684-1686.

7 Santi PA. J Histochem Cytochem 2011; 59:129-138.

8 Wang W, Fang H, Groom L, et al. Cell 2008; 134:279-290.

9 Shen EZ, Song CQ, Lin Y, et al. Nature 2014; 508:128-132.

10 Shang W, Lu F, Sun T, et al. Circ Res 2014; 114:412-420.
(Supplementary information is linked to the online version of the paper on the Cell Research website.)

(c) (i)(-) $\odot$ This work is licensed under the Creative Commons Attribution-NonCommercial-No Derivative Works 3.0 Unported License. To view a copy of this license, visit http:// creativecommons.org/licenses/by-nc-nd/3.0 